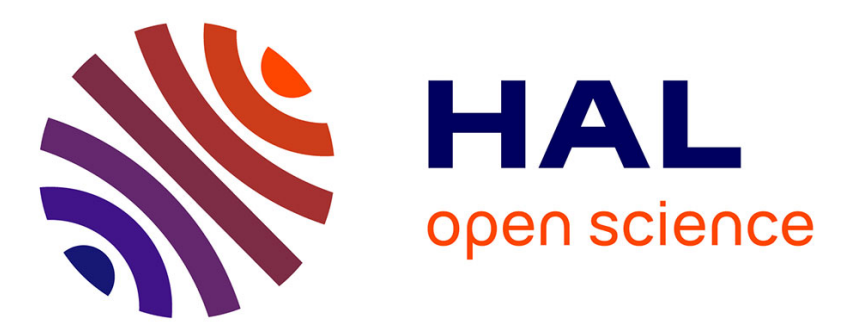

\title{
Nouveautés dans le traitement pharmacologique du choc cardiogénique
}

Valentine Léopold, Etienne Gayat

\section{To cite this version:}

Valentine Léopold, Etienne Gayat. Nouveautés dans le traitement pharmacologique du choc cardiogénique. Praticien en Anesthésie Réanimation, 2019, 23, pp.19 - 24. 10.1016/j.pratan.2018.12.002 . hal-03486449

\section{HAL Id: hal-03486449 \\ https://hal.science/hal-03486449}

Submitted on 20 Dec 2021

HAL is a multi-disciplinary open access archive for the deposit and dissemination of scientific research documents, whether they are published or not. The documents may come from teaching and research institutions in France or abroad, or from public or private research centers.
L'archive ouverte pluridisciplinaire HAL, est destinée au dépôt et à la diffusion de documents scientifiques de niveau recherche, publiés ou non, émanant des établissements d'enseignement et de recherche français ou étrangers, des laboratoires publics ou privés.

\section{다)(1) $(5$}

Distributed under a Creative Commons Attribution - NonCommercial| 4.0 International 


\section{Nouveautés dans le traitement pharmacologique du choc cardiogénique Update in pharmacological treatment of the cardiogenic shock}

Valentine LEOPOLD ${ }^{1,2}$, Etienne GAYAT $T^{1,2}$

1 Département d'Anesthésie - Réanimation, Hôpitaux Universitaires Saint Louis Lariboisière - Fernand Widal, Université Paris Diderot, Paris, France

${ }^{2}$ Biomarkers in CardioNeuroVascular diseases, UMR-S 942, Inserm, Paris, Fran

Auteur correspondant: Dr Etienne GAYAT

Département d'Anesthésie - Réanimation

Hôpitaux Universitaires Saint Louis - Lariboisière - Fernand Widal

Biomarlers in CArdio-Neuro-VAScular disesases, UMR-S 942, Inserm

2 rue Ambroise Paré

75010 Paris

Tel : 0149958071

Email : etienne.gayat@aphp.fr

Résumé : Le choc cardiogénique est un état d'hypoperfusion associée à un débit cardiaque altéré dont le traitement pharmacologique repose sur l'association de vasopresseurs et d'inotropes. Malgré les effets bénéfiques sur la contractilité, les inotropes ont des effets indésirables. De nouvelles molécules (Levosimendan, Milrinone, Enomixone, Ivabradine) prometteuses par leur mode d'action semblent pallier certains de ces défauts. Par ailleurs, les molécules historiques (Dobutamine, Noradrénaline, Adrénaline) font et feront l'objet de nouvelles études dans le choc cardiogénique, et leur utilisation est amenée à changer.

Mots clés : Choc cardiogénique, inotrope, vasopresseurs.

Abstract : Cardiogenic shock is a state of end organ hypoperfusion, associated with low cardiac output. The combination of inotropes and vasopressors is the goldstandard of cardiogenic shock pharmaceutical treatment. Despite beneficial effect on myocardial contractility, inotropes have inherent side effects. Recent studies have investigated the safety of these agents (Dobutamine, Norepinephrine, Epinephrine) and compared their 
efficacy. New promising molecules (Levosimendan, Milrinon, Enomixone, Ivabradine) have also been introduced; but they are used only occasionally.

Keywords: Cardiogenic shock, inotropes, vasopressors.

\section{Introduction}

Le choc cardiogénique est un état d'hypoperfusion sévère, associé à un débit cardiaque altéré, malgré une volémie optimisée. Son étiologie est dans 30 à $60 \%$ des cas l'insuffisance coronaire(1). Les recommandations publiées par l'European Society of Cardiology (ESC) (2) et les très récents avis d'experts (3) mettent en avant la multiplicité des axes de traitement du choc cardiogénique. En effet, la stratégie thérapeutique doit jouer sur trois différents tableaux : étiologique (revascularisation coronaire si nécessaire) pharmacologique (maintien hémodynamique) et mécanique (assistance circulatoire mécanique) (Tableau 1).

Le traitement pharmacologique du choc cardiogénique associe des agents vasoconstricteurs, généralement nécessaires pour maintenir une pression de perfusion et des agents inotropes, pour optimiser la contractilité myocardique. Pirracchio et coll. ont par ailleurs montré dans une population de 988 patients en choc cardiogénique que l'association d'un vasoconstricteur et d'un inotrope était associée à une meilleure survie (4). En pratique courante, les thérapeutiques pharmacologiques "classiques" sont encore les plus fréquemment utilisées: la noradrénaline et l'adrénaline comme agents principalement vasopresseurs, associée à la dobutamine comme inotrope (5).

Les "nouvelles " thérapeutiques, apparues ces dix dernières années, s'appuient sur des bases physiologiques intéressantes, et pallient les effets indésirables des inotropes classiques. Elles peinent cependant à trouver leur place dans le traitement du choc cardiogénique, malgré une littérature désormais abondante. Dans cette revue, nous traiterons des nouvelles thérapeutiques pharmacologiques, et leur place par rapport aux molécules classiques.

\section{Inotropes}

Les inotropes sont utilisés pour améliorer le débit cardiaque, en renforçant les fonctions myocardiques contractiles. Bien que nombre d'inotropes améliorent les paramètres hémodynamiques, les études successives peinent à montrer une amélioration en terme de mortalité. Cela vient probablement du fait que la majorité de ces molécules agissent sur la concentration de calcium intracellulaire et donc la contractilité myocardique, ce qui favorise aussi les arythmies sévères et la mort cellulaire (Figure 1) (6,7). D'autre part, l'entrée de calcium dans la cellule myocardique étant ATP dépendante, ces molécules augmentent la consommation myocardique en oxygène (8).

Dobutamine. II s'agit d'une catécholamine de synthèse utilisée depuis 40 ans. Elle est agoniste $\beta 1$ et $\beta 2$ ce qui lui confère un effet inotrope et vasodilatateur (9). Elle permet une augmentation du débit cardiaque, et une diminution des pressions de remplissage gauche. C'est aussi la seule catécholamine qui maintient un flux coronarien adapté à la consommation myocardique en oxygène par une vasodilatation coronaire (10). Elle est 
considérée comme référence dans le spectre des inotropes, mais son utilisation est limitée par son effet tachycardant et hypotenseur à la phase initiale du traitement.

Levosimendan. Mise sur le marché il y a maintenant 15 ans, cette molécule a montré son efficacité sur la mortalité à court-terme dans l'insuffisance cardiaque aiguë chez les patients sous bêta-bloquant (11). Cependant, dans les grandes cohortes et essais cliniques, les patients en choc cardiogénique étaient exclus ou sous-représentés. En présence de levosimendan, la troponine- $\mathrm{C}$ a une affinité accrue au calcium intracellulaire, ce qui améliore les performances contractiles de la troponine, sans modification du pool calcique intracellulaire. L'effet inotrope du levosimendan se fait donc sans augmentation de la consommation myocardique en oxygène (Figure 1). II a également été montré qu'à l'état stable le levosimendan a un effet de pléiotropie sur les cellules myocardique (12).

Milrinone et Enoximone. Ce sont des inhibiteurs de la phosphodiestérase III (PDEIII) (Figure 1), qui ont pour effet d'augmenter l'AMPc intracellulaire et donc l'influx de calcium dans les cellules myocardiques et dans les cellules musculaires lisses vasculaires. Leur effet est moins tachycardant que la dobutamine mais plus vasodilatateur et donc plus hypotenseur (13). Les études les comparant à la dobutamine portaient sur des patients souffrant d'insuffisance cardiaque décompensée. Les patients en choc cardiogénique étant exclus, ces résultats sont difficilement extrapolable à cette sous population $(13,14)$.

\section{Vasopresseurs}

Les agonistes adrénergiques sont les vasopresseurs de première ligne en raison de leur début d'action rapide, de leur puissance élevée et de leur demi-vie courte, ce qui permet un ajustement facile de la dose. La stimulation de chaque type de récepteur adrénergique a des effets potentiellement bénéfiques et nocifs. Par exemple, la $\beta$ stimulation adrénergique peut augmenter le débit sanguin, mais aussi le risque d'ischémie myocardique en raison de l'augmentation de la fréquence cardiaque et de la contractilité.

Parmi les catécholamines vasopressives, la molécule de référence reste la Noradrénaline. Elle a surtout des propriétés $\alpha$ adrénergiques, mais ses modestes effets $\beta$ adrénergiques aident à maintenir le débit cardiaque. L'administration entraîne généralement une augmentation, cliniquement significative, de la pression artérielle moyenne, avec peu de changement de la fréquence cardiaque ou du débit cardiaque.

La dopamine a un effet variable selon la posologie, ce qui la rend difficilement maniable. Elle a longtemps été utilisée à fortes doses comme vasopresseur, et à faible doses pour un effet supposé protecteur rénal qui a fini par être infirmé par de nombreuses études et méta-analyses successives (15). Dans un essai clinique randomisé contrôlé, comparant noradrénaline et dopamine, l'analyse du sous-groupe de 280 patients en choc cardiogénique a révélé une surmortalité des patients traités par dopamine (16). Son utilisation a ensuite progressivement diminué dans cette indication (17) et n'est plus recommandée à ce jour.

L'adrénaline a longtemps gardé une place en pratique courante dans le traitement du choc cardiogénique, de par son double effet inotrope et vasopresseur. De récentes études apportent désormais de solides preuves que la noradrénaline est supérieure à 
l'adrénaline dans cette indication. En particulier, la seule étude randomisée contrôlée comparant l'adrénaline à la noradrénaline dans le choc cardiogénique a montré une nette supériorité de la noradrénaline sur l'incidence de choc réfractaire, bien que les performances cardiaques soient similaires dans les deux groupes (18). Les patients traités par adrénaline présentent également des effets secondaires néfastes comme l'hyperglycémie et l'acidose métabolique via la stimulation $\beta 2$-adrénergique, et la tachycardie (19).

Par ailleurs, la méta-analyse des données individuelles de 2579 patients en choc cardiogénique, montre une surmortalité importante des patients traités par adrénaline par rapport à ceux traités par toute autre association de traitements (Figure 2B) (20).

\section{Contrôle de la fréquence cardiaque}

L'ivabradine est un inhibiteur des canaux $\mathrm{Na} / \mathrm{K}$ des cellules du noeud sino-atrial, ce qui lui confère un effet chronotrope négatif pur, sans effet inotrope. Le rationnel de l'utilisation d'un agent bradycardisant dans le choc cardiogénique se base sur l'effet Bowditch, qui explique pourquoi la tachycardie induite par les inotropes diminue la puissance contractile myocardique: pendant la diastole, le $\mathrm{Ca}^{++}$intracellulaire est efflué par la pompe $3 \mathrm{Na}^{+} / \mathrm{Ca}^{++}$avant la prochaine systole. L'influx de $\mathrm{Na}^{+}$est contrebalancé par la $\mathrm{Na}^{+} / \mathrm{K}^{+}$-ATPase. Lorsque la fréquence cardiaque s'accélère, la diastole se raccourcit, la pompe $3 \mathrm{Na}^{+} / \mathrm{Ca}^{++}$est en activité maximale et la $\mathrm{Na} / \mathrm{K}+$ ATPase $\mathrm{n}^{\prime}$ arrive plus à maintenir le rythme de l'efflux de $\mathrm{Nat}$, et ralentit alors la cadence de l'efflux calcique (effet Bowditch) (21). La preuve de l'effet bénéfique de la réduction de la fréquence cardiaque sur les performances myocardiques a été réalisée par Gallet et coll. sur 9 patients en choc cardiogénique réfractaire (22). Dans cette étude, l'ajout d'ivabradine à la dobutamine et à la noradrénaline permettait non seulement une amélioration des paramètres hémodynamiques mais aussi des signes cliniques d'hypoperfusion ( $\mathrm{SvO} 2$, diurèse). Cette première étude pilote a été suivie de deux études randomisées contrôlées actuellement en cours l'une américaine l'autre espagnole, comparant l'ajout d'ivabradine au traitement standard chez des patients en choc cardiogénique. Rendez-vous en 2019. Par ailleurs l'ivabradine n'est actuellement commercialisé que sous forme orale.

\section{En pratique : que choisir?}

De manière générale, l'association d'un vasopresseur et d'un inotrope est préférable à I'utilisation d'un vasopresseur seul, en particulier si le volume d'éjection systolique, le débit cardiaque (4). D'autre part, les agents à effet pur (noradrénaline, dobutamine) devraient être privilégier par rapport aux agents à effet combiné (adrénaline, dopamine) pour une meilleure titration des effets hémodynamiques et inotropes attendus.

Une méta-analyse récente de la Cochrane Library a comparé les différents inotropes en termes de mortalité à court terme dans le choc cardiogénique (23). Les études randomisées contrôlées de bonne qualité portant sur des patients en choc cardiogénique sont rares, mais il semble que le levosimendan et la dobutamine aient des effets similaires dans cette indication, sur la mortalité à court terme dans le choc cardiogénique (niveau de preuve bas) (Figure 2A). Une autre méta-analyse portant exclusivement sur les patients de chirurgie 
cardiaque retrouvait le même résultat (24). L'utilisation du levosimendan en France est possible depuis peu.

Concernant les vasopresseurs, la noradrénaline doit être préférée à l'adrénaline. L'adrénaline et la dopamine devraient être abandonnées dans le choc cardiogénique car ces molécules sont délétères en termes de mortalité (Figure 2B).

Les recommandations de l'European Society of Cardiology (ESC) 2016 sur la prise en charge du choc cardiogénique (Tableau 1) vont dans ce sens : la noradrénaline doit être utilisée en priorité, après restauration d'une euvolémie. Si l'utilisation d'un inotrope est nécessaire, la dobutamine fait référence. Les inhibiteurs de phosphodiestérase III et le levosimendan ont une place marginale : il est recommandé de les utiliser chez les patients dont l'hypotension est en partie imputable à un traitement bêtabloquant, pour qui les catécholamines seraient inefficaces sur l'inotropisme. Enfin, ces recommandations ne retiennent pas d'indication à la dopamine ni à l'adrénaline (2).

Un support circulatoire mécanique temporaire est recommandé en seconde intention, en cas de choc réfractaire au traitement médical, selon les comorbidités du patient. Le Ballon de contre pulsion intra-aortique (CPIA) n'est pas recommandé, devant l'absence d'efficacité démontré dans un essai randomisant 600 patients en choc cardiogénique post-infarctus (CPIA versus soins courants) (25). 
Tableau 1. Traduction des Recommandations ESC 2016 sur la prise en charge du choc cardiogénique (2).

\begin{tabular}{|c|c|c|}
\hline Adapté des Recommandations ESC 2016 & Classe & Niveau \\
\hline $\begin{array}{l}\text { Tout patient en état de choc cardiogénique doit bénéficier immédiatement d'un ECG et d'une } \\
\text { echocardiographie. }\end{array}$ & 1 & C \\
\hline $\begin{array}{l}\text { Tout patient en état de choc cardiogénique doit être rapidement transféré vers un centre } \\
\text { tertiaire disposant d'une salle de coronarographie disponible, et d'un service de soins continu } \\
\text { cardiologique ou réanimation spécialisée, en mesure de proposer un support circulatoire } \\
\text { mécanique dans de brefs délais. }\end{array}$ & 1 & C \\
\hline $\begin{array}{l}\text { Chez les patients en état de choc cardiogénique compliquant un syndrome coronarien aigu, il } \\
\text { est recommandé de réaliser une coronarographie pour revascularisation coronaire dans un } \\
\text { délai de } 2 \mathrm{~h} \text { après admission. }\end{array}$ & 1 & C \\
\hline Un monitorage continu de l'ECG et de la pression artérielle sont recommandés. & 1 & C \\
\hline Un monitorage invasif de la pression artérielle est recommandé. & I & $\mathrm{C}$ \\
\hline $\begin{array}{l}\text { II est recommandé de réaliser un test de remplissage }(>200 \mathrm{ml} / 15 \mathrm{~min} \text { de Sérum Salé } \\
\text { Isotonique ou Ringer Lactate) en première intension, en l'absence de signe de surcharge. }\end{array}$ & 1 & $\mathrm{C}$ \\
\hline Un traitement inotrope (Dobutamine) peut être envisagé pour améliorer le débit cardiaque. & $\mathrm{Ilb}$ & C \\
\hline $\begin{array}{l}\text { Un traitement vasopresseur (noradrénaline à préférer à la dopamine) peut être envisagé, en } \\
\text { cas d'hypotension artérielle associés à des signes d'ypoperfusion persistants. }\end{array}$ & Illb & C \\
\hline La CPIA n'est pas recommandée en routine. & III & $\mathrm{C}$ \\
\hline $\begin{array}{l}\text { Un support circulatoire mécanique temporaire peut être envisagé en cas de choc } \\
\text { cardiogénique réfractaire, selon l'age, les comorbidités et les fonctions cognitives du patient. }\end{array}$ & Illb & $\mathrm{C}$ \\
\hline
\end{tabular}

ECG : electro-cardio-gramme. CPIA : contre-pulsion intra aortique 


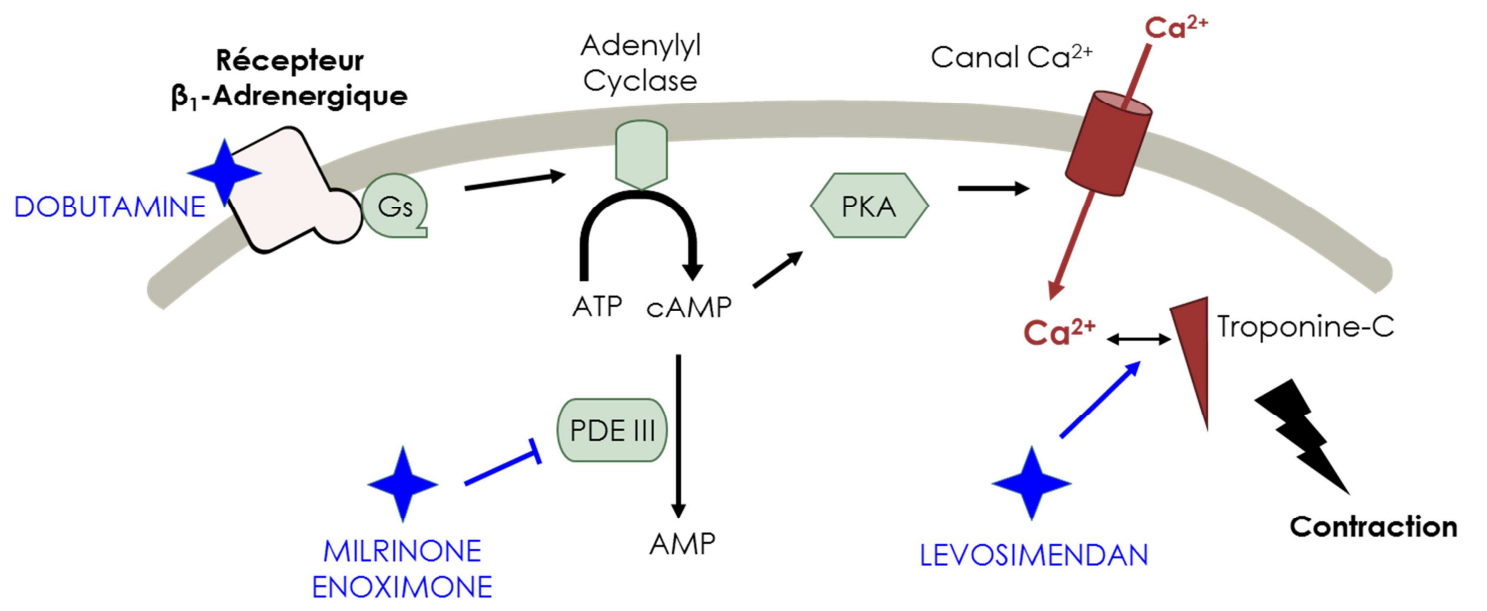

Figure 1 : Mécanisme d'action des principaux inotropes. (6-8)

La dobutamine est un agoniste des récepteurs $\beta 1$ adrénergique présents à la surface des cardiomyocytes. Leur activation entraine l'activation de l'adenylyl cyclase via les protéines $G$ stimulatrices (Gs). L'adenylyl cyclase catalyse la conversion d'adénosine triphosphate (ATP) en adénosine mono phosphate cyclique (cAMP). L'augmentation de cAMP intracellulaire active la Protéine Kinase $A$ (PKA) ce qui a pour effet d'ouvrir les canaux calciques membranaires, permettant une entrée de calcium dans la cellule. La liaison du calcium à la troponine est nécessaire en systole pour la contraction myocardique. L'AMP est convertit en AMP par la phosphodiestérase III (PDE III). Les inhibiteurs de la PDEIII (Milrinone, Enoximone) bloquent cette enzyme ce qui a pour conséquence une augmentation d'AMPc, et une concentration en calcium intracellulaire accrue à l'origine de l'effet inotrope positif. Le levosimendan lui est un sensibilisateur calcique de la troponine- $C$ et augmente la contractilité, sans modifier le pool intracellulaire de calcium. 

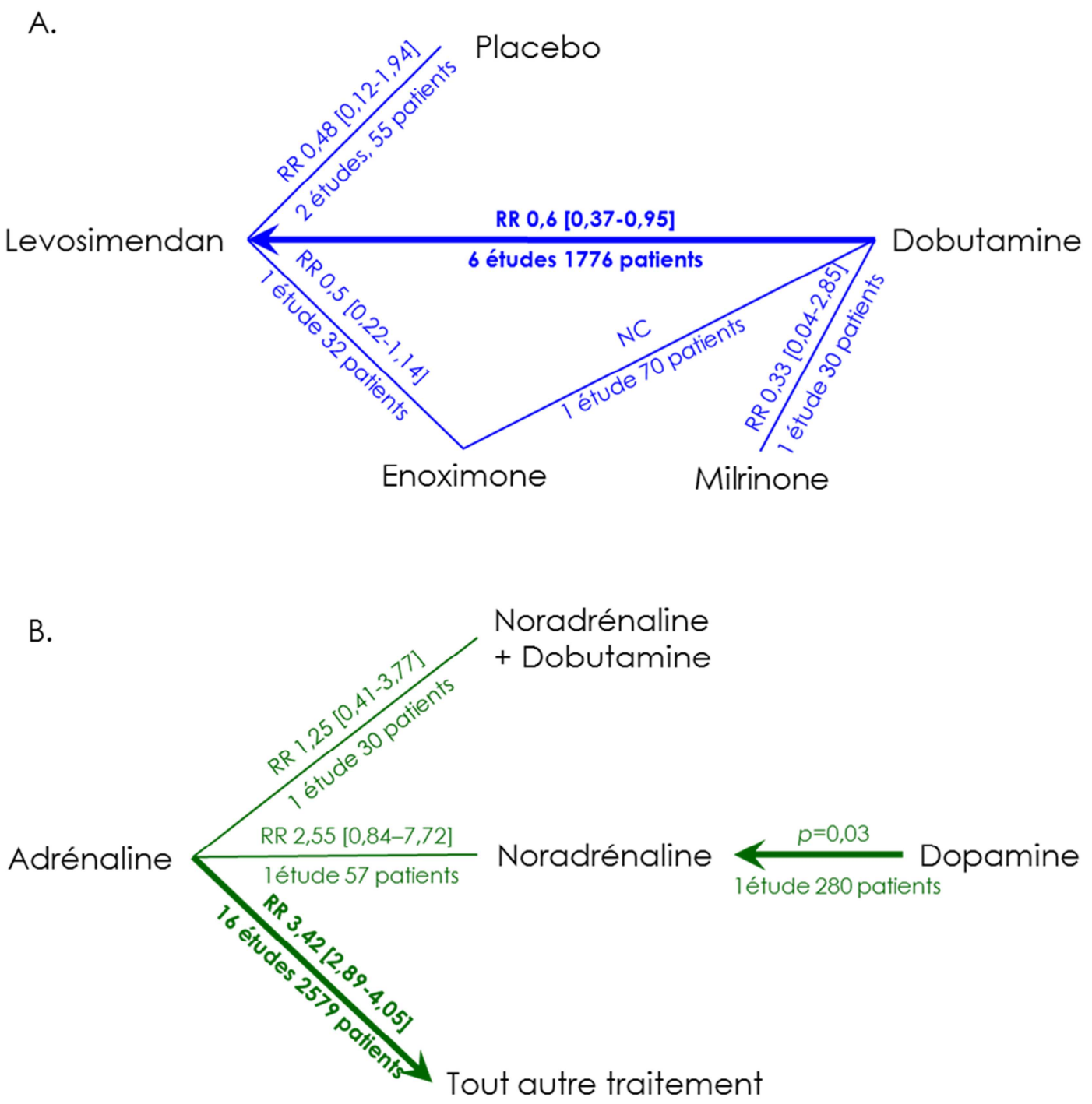

Figure 2: Comparaison des inotropes ( $A$, d'après (23)) et vasopresseurs ( $B$, d'après $(16,18,20,26))$ sur la mortalité à court terme.

Les liens entre deux molécules représentent l'existence d'essai(s) randomisé(s) contrôlé(s) comparant directement les deux molécules sur la mortalité à court terme; à l'exception de la comparaison entre " Adrénaline » et " Tout autre traitement " qui est le fruit d'une métaanalyse sur données individuelles et score de propension sur 16 études (20), et non d'une comparaison directe.

Les liens en gras indiquent une différence significative, les flèches pointent vers la molécule ayant montré sa supériorité.

Le Risque Relatif (RR [IC 95\%]) de mortalité à court terme, le nombre d'études concernées et le nombre de patients comparés sont indiqués pour chaque comparaison. Lorsqu'il y a plus d'une étude, le RR est celui de méta-analyses publiées $(20,23)$. NC : mortalité non rapportée. 


\section{Bibliographie}

1. Prondzinsky $R$, Unverzagt $S$, Russ $M$, Lemm $H$, Swyter $M$, Wegener $N$, et al. Hemodynamic effects of intra-aortic balloon counterpulsation in patients with acute myocardial infarction complicated by cardiogenic shock: the prospective, randomized IABP shock trial. Shock. 2012 Apr;37(4):378-84.

2. Ponikowski P, Voors AA, Anker SD, Bueno H, Cleland JGF, Coats AJS, et al. 2016 ESC Guidelines for the diagnosis and treatment of acute and chronic heart failure. Eur Heart J. 2016;37(27):2129-2200m.

3. Mebazaa A, Combes A, van Diepen S, Hollinger A, Katz JN, Landoni G, et al. Management of cardiogenic shock complicating myocardial infarction. Intensive Care Medicine. 2018;1-14.

4. Pirracchio R, Parenica J, Resche Rigon M, Chevret S, Spinar J, Jarkovsky J, et al. The Effectiveness of Inodilators in Reducing Short Term Mortality among Patient with Severe Cardiogenic Shock: A Propensity-Based Analysis. PLoS One. 2013;8(8):1-10.

5. Zannad F, Mebazaa A, Juilliere Y, Cohen-Solal A, Guize L, Alla F, et al. Clinical profile, contemporary management and one-year mortality in patients with severe acute heart failure syndromes: The EFICA study. Eur J Heart Fail. 2006 Nov;8(7):697-705.

6. Bers DM. Cardiac excitation-contraction coupling. Nature. 2002 Jan;415(6868):198205.

7. McBride BF, White CM. Acute decompensated heart failure: a contemporary approach to pharmacotherapeutic management. Pharmacotherapy. 2003 Aug;23(8):997-1020.

8. Arrigo M, Mebazaa A. Understanding the differences among inotropes. Intensive Care Med. 2015 May;41(5):912-5.

9. Akhtar N, Mikulic E, Cohn JN, Chaudhry MH. Hemodynamic effect of dobutamine in patients with severe heart failure. Am J Cardiol. 1975 Aug;36(2):202-5.

10. Fowler MB, Alderman EL, Oesterle SN, Derby G, Daughters GT, Stinson EB, et al. Dobutamine and dopamine after cardiac surgery: greater augmentation of myocardial blood flow with dobutamine. Circulation. 1984 Sep;70(3 Pt 2):I103-11.

11. De Luca L, Colucci WS, Nieminen MS, Massie BM, Gheorghiade M. Evidence-based use of levosimendan in different clinical settings. Eur Heart J. 2006 Aug;27(16):1908-20.

12. Farmakis D, Alvarez J, Gal T Ben, Brito D, Fedele F, Fonseca C, et al. Levosimendan beyond inotropy and acute heart failure: Evidence of pleiotropic effects on the heart and other organs: An expert panel position paper. Int J Cardiol. 2016 Nov;222:303-12.

13. Felker GM, Benza RL, Chandler AB, Leimberger JD, Cuffe MS, Califf RM, et al. Heart failure etiology and response to milrinone in decompensated heart failure: results from the OPTIME-CHF study. J Am Coll Cardiol. 2003 Mar;41(6):997-1003.

14. Abraham WT, Adams KF, Fonarow GC, Costanzo MR, Berkowitz RL, LeJemtel TH, et al. In-hospital mortality in patients with acute decompensated heart failure requiring intravenous vasoactive medications: an analysis from the Acute Decompensated Heart Failure National Registry (ADHERE). J Am Coll Cardiol. 2005 Jul;46(1):57-64. 
15. Joannidis M, Druml W, Forni LG, Groeneveld ABJ, Honore PM, Hoste E, et al. Prevention of acute kidney injury and protection of renal function in the intensive care unit: update 2017 : Expert opinion of the Working Group on Prevention, AKI section, European Society of Intensive Care Medicine. Intensive Care Med. 2017 Jun;43(6):730-49.

16. De Backer D, Biston P, Devriendt J, Madl C, Chochrad D, Aldecoa C, et al. Comparison of dopamine and norepinephrine in the treatment of shock. N Engl J Med. 2010 Mar;362(9):779-89.

17. Kon ZN, Bittle GJ, Pasrija C, Pham SM, Mazzeffi MA, Herr DL, et al. Venovenous Versus Venoarterial Extracorporeal Membrane Oxygenation for Adult Patients With Acute Respiratory Distress Syndrome Requiring Precannulation Hemodynamic Support: A Review of the ELSO Registry. Ann Thorac Surg. 2017 Aug;104(2):645-9.

18. Levy B, Clere-Jehl R, Legras A, Morichau-Beauchant T, Leone M, Frederique G, et al. Epinephrine Versus Norepinephrine for Cardiogenic Shock After Acute Myocardial Infarction. J Am Coll Cardiol. 2018 Jul;72(2):173-82.

19. Harjola V-P, Lassus J, Sionis A, Køber L, Tarvasmäki T, Spinar J, et al. Clinical picture and risk prediction of short-term mortality in cardiogenic shock. Eur J Heart Fail. 2015;17:501-9.

20. Leopold V, Gayat E, Pirracchio R, Spinar J, Parenica J, Tarvasmaki T, et al. Epinephrine and short-term survival in cardiogenic shock: an individual data meta-analysis of 2583 patients. Intensive Care Med. 2018 Jun;44(6):847-56.

21. Ward JP, Linden RW. Physiology at a glance. Igarss 2014. 2014. 1-5 p.

22. Gallet R, Ternacle J, Damy T, Guendouz S, Bremont C, Seemann A, et al. Hemodynamic effects of Ivabradine in addition to dobutamine in patients with severe systolic dysfunction. Int J Cardiol. 2014 Sep;176(2):450-5.

23. Schumann J, Henrich EC, Strobl H, Prondzinsky R, Weiche S, Thiele H, et al. Inotropic agents and vasodilator strategies for the treatment of cardiogenic shock or low cardiac output syndrome. Cochrane database Syst Rev. 2018 Jan;1:CD009669.

24. Belletti A, Castro ML, Silvetti S, Greco T, Biondi-Zoccai G, Pasin L, et al. The Effect of inotropes and vasopressors on mortality: a meta-analysis of randomized clinical trials. Br J Anaesth. 2015 Nov;115(5):656-75.

25. Thiele H, Zeymer U, Neumann F-J, Ferenc M, Olbrich H-G, Hausleiter J, et al. Intraaortic Balloon Support for Myocardial Infarction with Cardiogenic Shock. N Engl J Med [Internet]. 2012;367(14):1287-96. Available from: http://www.nejm.org/doi/10.1056/NEJMoa1208410

26. Levy B, Perez P, Perny J, Thivilier C, Gerard A. Comparison of norepinephrinedobutamine to epinephrine for hemodynamics, lactate metabolism, and organ function variables in cardiogenic shock. A prospective, randomized pilot study. Crit Care Med [Internet]. 2011;39(3):450-5. Available from: http://content.wkhealth.com/linkback/openurl?sid=WKPTLP:landingpage\&an=00003 246-201103000-00004 